\title{
Numerical modeling of induction heating systems with load of azimuthal periodicity
}

\author{
Emil Mannanov*, Sergei Galunin and Alexey Shatunov \\ St. Petersburg Electrotechnical University "LETI"
}

\begin{abstract}
The paper presents developed numerical modelling methodology of induction heating problems of disks with a simple and complex profile in 3D for calculating the coupled electromagnetic and thermal tasks in the parametric design language (ANSYS APDL). We demonstrate that the developed tools can be used for products of various sizes, as well as for other metal engineering components using the example of local electromagnetic processing of a metal disk and a diaphragm spring. The authors used development for parametric research and design of induction heaters. The described methodology has particular specificity, due to which it is possible to expand the scope for a number of engineering units with azimuthal periodicity property.
\end{abstract}

\section{Introduction}

The classical way of the numerical realization of motion during induction processing is to shift the inductor, relative to a stationary object of heating. In this case, the inductor step by step describes the trajectory of the proposed movement. The nature of the movement determines the type of shift. In the case of rotation, shift is movement by a predetermined number of degrees. Each new position of the inductor induces an array of heat sources, saving the simulation results of all previous iterations.

In [8], the angular velocity $(\mathrm{Hz}, \mathrm{r} / \mathrm{s})$ described in the global Cartesian coordinate system X, Y and Z-axis are set as constants: OMEGAX, OMEGAY, OMEGAZ. The parameters XLOC, YLOC, ZLOC describe the location of the axes of the rotating body in the $\mathrm{X}, \mathrm{Y}$, and $\mathrm{Z}$ directions, respectively.

In this paper, the authors replaced the rotation by setting strongly nonlinear properties of the material. This solution has a limitation associated with the distortion of the known electrothermal properties of the heated material at high rotation speeds. Data are known when the rotation speed can make a significant contribution to increasing the efficiency of induction heating $[1,4,12$, 15]. However, taking into account the results of [6], at a low speed of rotation of the metal disk (18 rpm), the final temperature distribution formed due to the processes of thermal material conductivity and heat losses from surfaces (confirmed experimentally). The temperature level of $450^{\circ} \mathrm{C}$ degrees based on the results of [5], which authors shows the data obtained after induction tempering a sample with $3 \mathrm{~mm}$ thick. The goal function - ensuring uniform induction processing of the diaphragm spring disk with a minimum temperature deviation from a given temperature level. The initial electrical parameters (current, frequency, heating time, disk diameters and the inductor design repeat the natural sample [6].

\section{Methods}

Within research, authors used a computer type of experiment. The numerical simulation environment is ANSYS APDL (ANSYS Parametric Design Language). The solution of the electromagnetic problem performed with a magnetic vector potential [7 - 12]. For solution, the three-dimensional finite electromagnetic problem we used SOLID97 element in order to increase the accuracy of calculation. The processes occurring in metals during the operation of an induction installation are described by a system of interconnected electromagnetism and thermal conductivity equations with non-linear coefficients. In the simulated system, nonlinear coefficients associate with the dependence of the metal products properties on temperature and electromagnetic field intensity, therefore the electromagnetic and thermal problems are related. To perform thermal transient analysis was used SOLID90 element. An algorithm for coupling solutions to the problems of electromagnetism and thermophysics were earlier presented and described in detail in $[2,14]$.

\section{The geometry}

There are several ways to accomplish this task. The most obvious are direct working with ANSYS graphical tools or preliminary preparation of a CAD model with the subsequent import of the finished geometry into a numerical environment. The third way is programming. As part of the study, the geometry of the system is

\footnotetext{
* Corresponding author: emil-mannanov@mail.ru
} 
implemented using the built-in programming language APDL.

The optimization parameters are the turn's width and the inductor height, the air gap, the pole pitch, the operating frequency, the current value, the current direction, the heating time, the diameter and thickness of the heating object, and the electrothermal nonlinear disk properties. In addition, the diaphragm spring dimensions shown in Fig. 1 are parameterized.

Geometry modeling also has a common feature associated with the possibility of using the symmetry of the system. The investigated system has this property, therefore, instead of modeling the complex geometry, authors decided to simulate the section using the azimuthal periodicity of the product structure. It allows us to adjust the number of fingers of the spring and reduce the time for calculations. The standard number of fingers placed around the circumference of the diaphragm spring is: $24,20,18$ and 12 . Summarize the data is in the Table 1.

Table 1.

\begin{tabular}{|c|c|c|c|c|}
\hline $\begin{array}{c}\text { Number of fingers of the } \\
\text { spring }\end{array}$ & 12 & 18 & 20 & 24 \\
\hline Modeling area & $15^{0}$ & $10^{0}$ & $9^{0}$ & $7^{0} 5^{\prime}$ \\
\hline
\end{tabular}

In fig. 1 shows a sketch of a diaphragm spring. The initial modelling area is equal to $9^{0}$. In fact, this means the basic spring design of the has 20 fingers. The estimated time required for calculation at a given accuracy can be minimized by 40 times. The total height of the unloaded disk is equal to the loaded. In other words, the product does not have the curvature necessary for operation and has a flat shape under load. During the heat treatment procedure performing, this aspect doesn't play a significant role, since to ensure the uniform local heating and the size of the heat treatment zone, the turns of the heater have to be located: 1 . on one axis; 2 . parallel to the plane of the heating zone. It allows adjusting the input power by changing the inductor current, the pitch of the turns and the air gap.

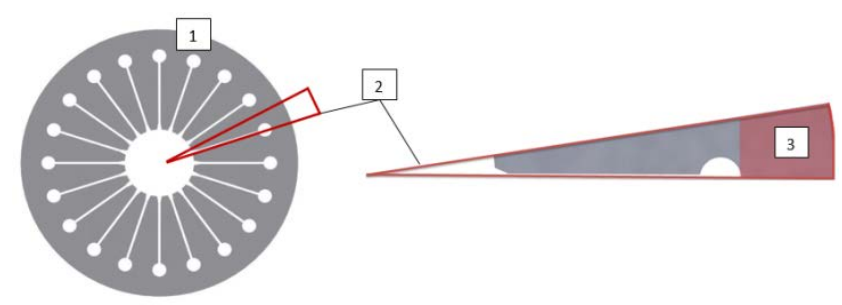

Fig. 1. Sketch of the investigated system: 1 - General view of the product; 2 - Modeling area; 3 - heating zone.

In the model presented in fig. 2a), is given a partition into the FE mesh. Grid nodes concentrated in the heating zone, which is able to provide model flexibility in parametric studies. The numerical solution of the coupled electrothermal problem requires the use of special grids due to the manifestation of the surface effect. Joule heat sources are generated in the layer which relatively small comparing to the total workpiece size, therefore this aspect needs to be taken into account and optimize to increase the modeling accuracy with the least computational and time costs. For the case of the disk modeling with a complex profile, the grid is optimized and has the prisms form of elements (see Fig. 2, b)). Similar to the experimental data [6], the inductor positioning relative to the diaphragm spring repeats fullscale samples.

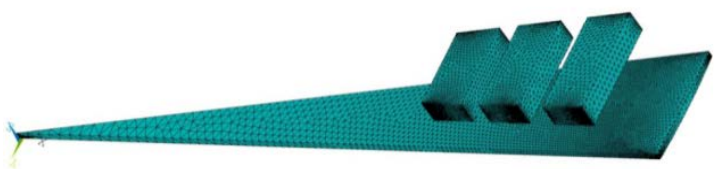

a)

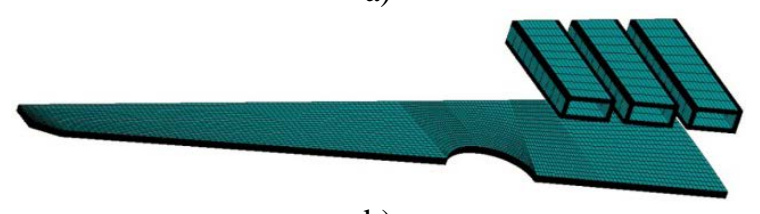

b)

Fig. 2. Finite-element 3D systems: a) "Inductor - disk"; b) "Inductor - diaphragm spring".

\section{Boundary conditions}

A zero value of the vector magnetic potential $\mathrm{A}=0$ is set at the external boundaries of the system. This means at the external boundary (air) remote from the Joule heat sources, the complete attenuation of the electromagnetic waves are assumed. The Neumann condition is accepted by default in the absence of specified Border conditions. The description of the homogeneous Neumann condition at the external boundary indicates the absence of the tangent component of magnetic flux.

A zero electric potential is set at the ends of the turns of the inductor and on the opposite boundary, the degree of freedom VOLT and the current value are set for the inductor nodes. It is also possible to set up the counter currents to simulate the induction transverse flux heating. The type of thermal analysis is transitional, boundary condition of the III kind To solve the thermal problem, the internal sources are reading from the electromagnetic calculation. The temperature selected as the initial conditions is 20 degrees.

\section{Results and Discussion}

It is most logical to evaluate the relatively simple system configuration "Inductor - Disk". Fig. 3 - 5 shows the obtained electrothermal solution to the problem. Modeling area is $9^{0}$. The problem statement corresponds to the experimental data [6] and the problem in which a spiral inductor located under a heated disk. The verification of computer simulation authors performed based on an analysis of the eddy currents distribution, specific power and temperature field over the surface of a heated disk.

It is worth noting, the calculation results in Figs. 3 and 4 indicate a correct description of the boundary conditions. Consider first of all Fig. 3. As you know, researchers and engineers design inductors taking into 


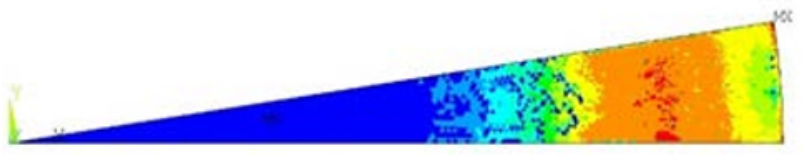

a)
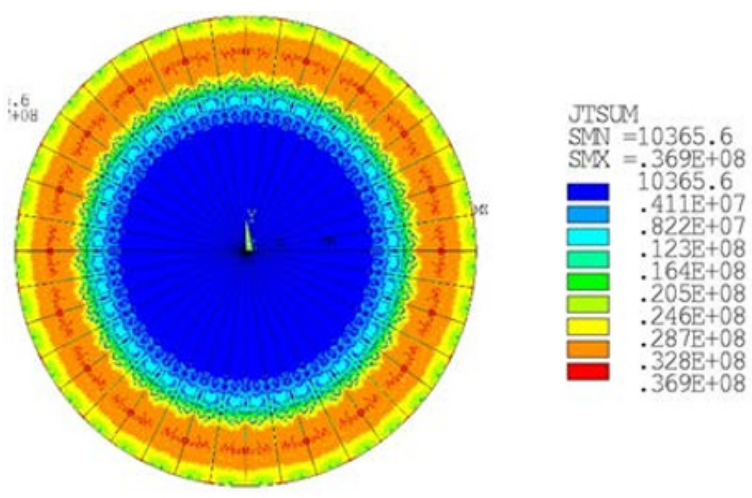

b)

Fig. 3. Total Current Density. a) Modeling area - $9^{0}$; b) General view.

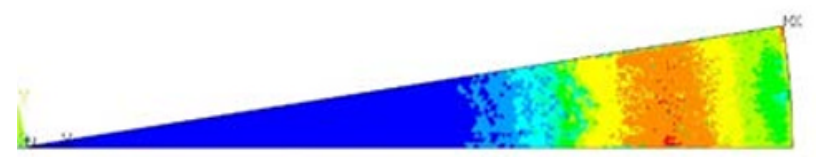

a)

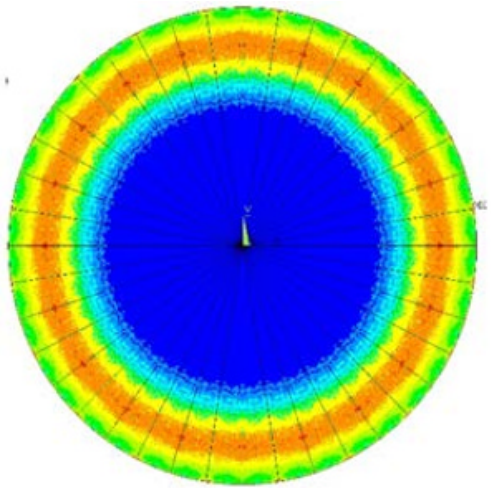

b)

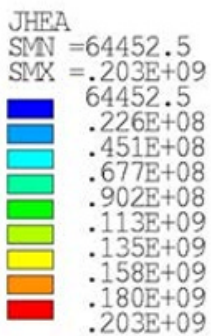

Fig. 4. Joule Heat Generation on the disk surface after 30 s heating. a) Modeling area - $9^{0}$; b) General view.

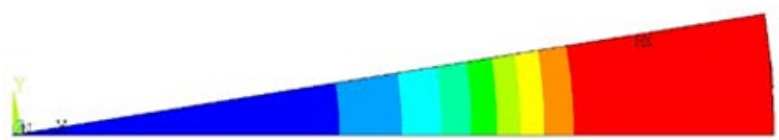

a)

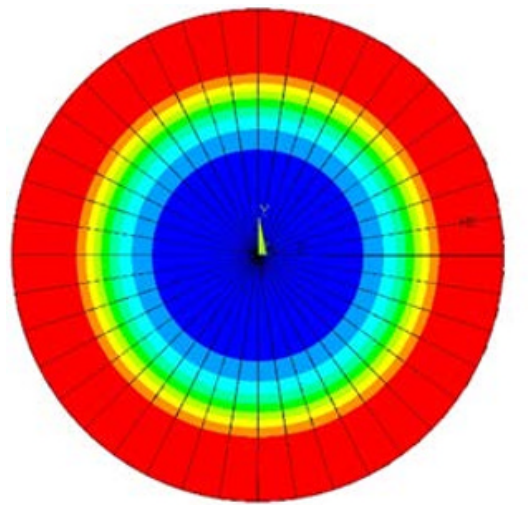

b)

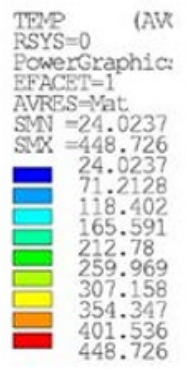

Fig. 5. The final temperature distribution on the disk surface after 30 s heating. a) Modeling area - $9^{0}$; b) General view.

account the physical phenomenon - the surface effect. Since the system in question has more than one turn, inhomogeneities in the current density distribution over the surface of the heaters occur as a result of a physical phenomenon called the proximity effect, which is a form of surface effect. Naturally, eddy currents in the disk repeat the inductor shape. A key feature of the solution is eddy currents circulate along the edge of the disk, which is clearly seen in Fig. 4. and 5 (the current density induced in the disk and Joule Heat Generation on the disk surface).

However, analyzing the final temperature field after the induction heating completion, the disk edges overheating doesn't observed and the maximum temperature on the surface shifted toward the center due to heat losses from the disk surface, which also corresponds to experiment [6]. In the next step, consider a disk with a complex profile. The problem statement is formulated similarly to the "Inductor - disk" system. 


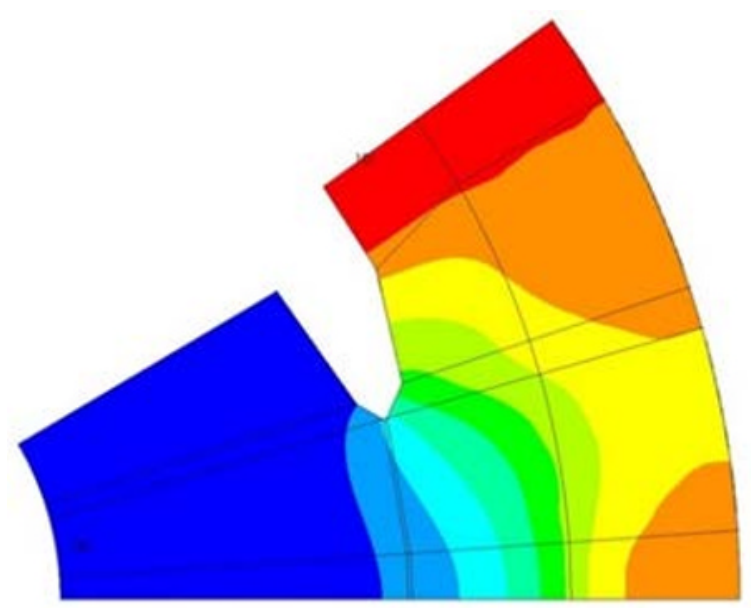

a)

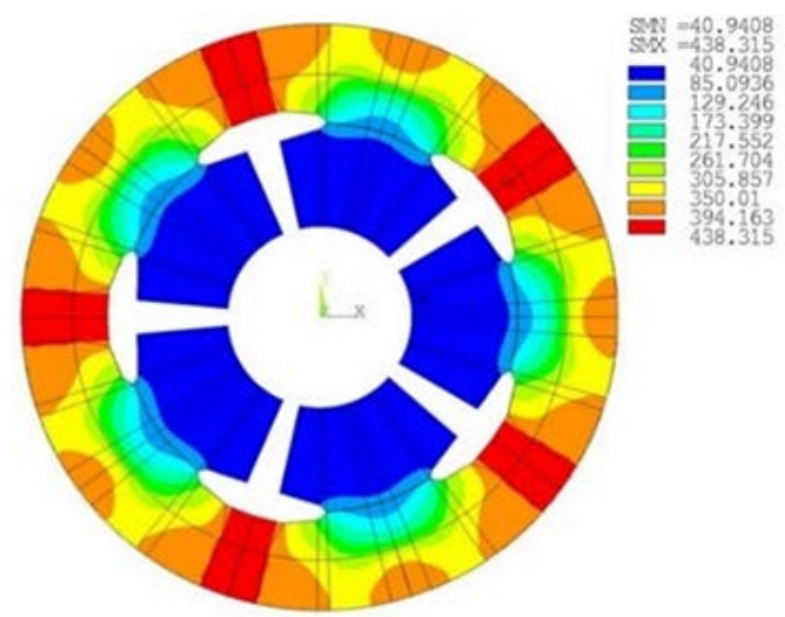

b)

Fig. 6. The final temperature distribution after the heating process completion in a longitudinal magnetic field with poor quality processing of the surface of the diaphragm spring in the area of the bend of the fingers. a) Modeling area - $36^{0}$; b) General view.

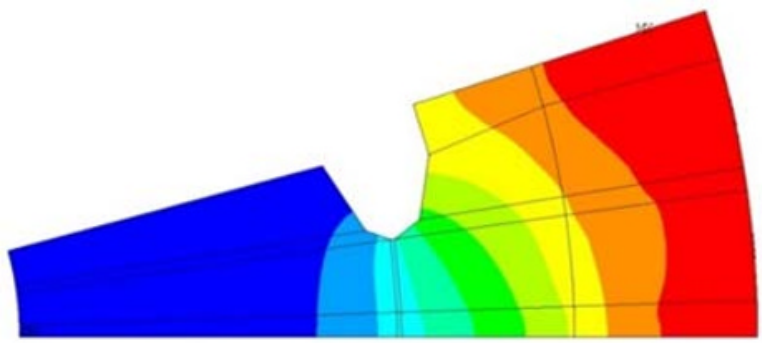

a)

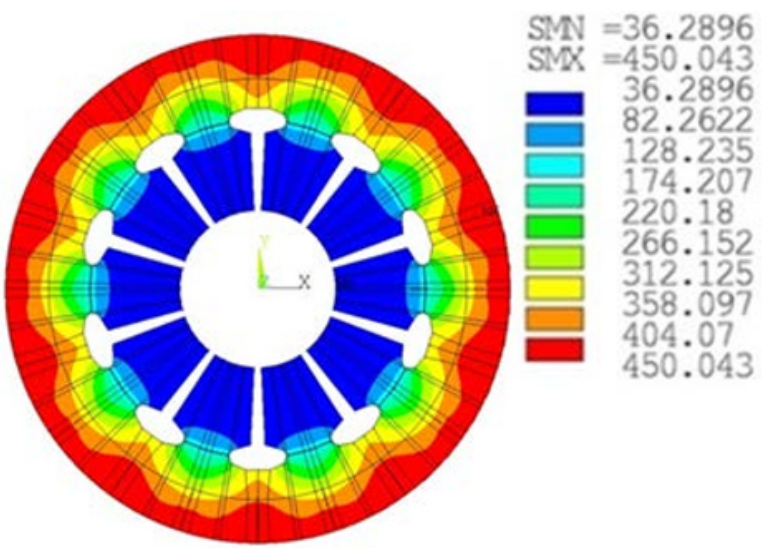

b)

Fig. 7. The final temperature distribution after the heating process completion in a longitudinal magnetic field with poor quality processing of the surface of the diaphragm spring in the area of the bend of the fingers. a) Modeling area - 18

Let's pretend that the system in the calculation area is: 1) $36^{0}$, 2) $18^{0}$; 3) $15^{0}$; 4) $7^{0} 5^{\prime}$ which correspond to 1) 5 ; 2) 10; 3) 12; 4) 24 fingers on the diaphragm spring. In fact, the number of fingers equal to 5 and 10 does not take place for a number of reasons. 12 fingers is the minimum number of fingers, and 24 is the maximum possible. A formulation of the modeling problem is capable of illuminating the limits of the approach under consideration.

The estimation of the correctness of the numerical solution is based on the analysis of the uniformity of the temperature distribution around the circumference of the heated diaphragm spring and comparison with the experimental results, as well as with data published in scientific journals. The first test electrothermal results are presented in Fig. 6.

Along the circumference of the outer diameter visually observed local temperature differences of up to $130{ }^{\circ} \mathrm{C}$. The sharp temperature inhomogeneity indicates the unnatural behavior of the temperature distribution, which evidences the selected computational modeling area is not applicable in practice. Consider the test results for option 2), shown in Fig. 7.

During analyzing the obtained results by narrowing the modeling area to 180 , we observed temperature differences along the circumference within acceptable limits. However, the final temperature distribution should be considered unsatisfactory. The final test for options 3) and 4) are presented in Fig. 8 and 9.

Developing problem-oriented models for induction disk heating systems by rotation in a high-frequency magnetic field, the minimum allowable calculation area for modeling in the same a formulation is an area not exceeding $15^{0}$. Modeling a diaphragm spring disk with the maximum number of fingers has the most uniform and close to experiment temperature distribution [6].

\section{Conclusions}

Two programs and a method for solving the coupled electrothermal problems of induction heating of machine-building units by numerical simulation in the 


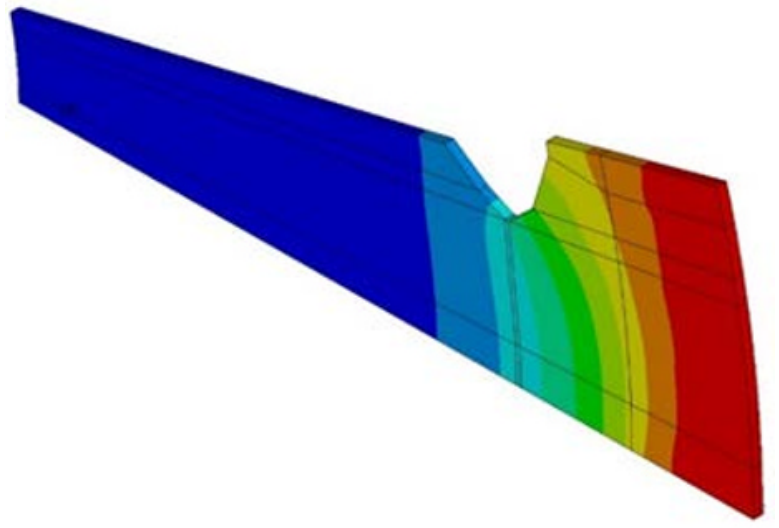

a)

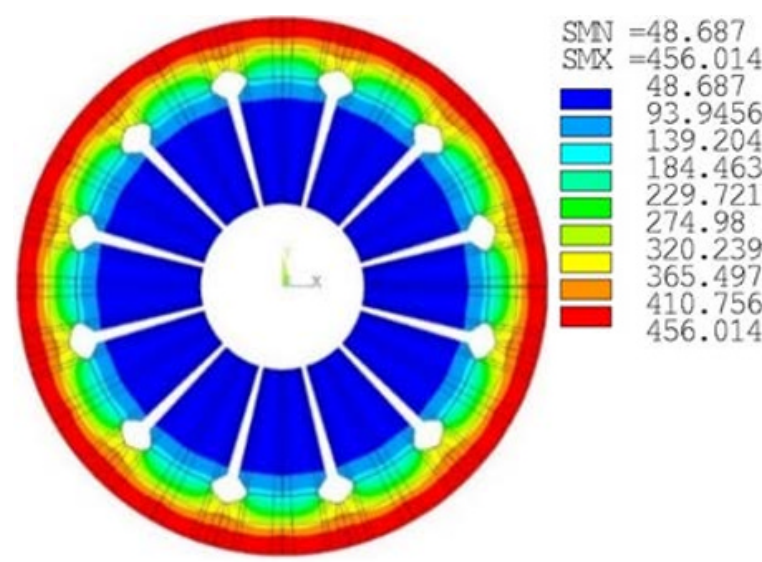

b)

Fig. 8. The final temperature distribution after the heating process completion in a longitudinal magnetic field with poor quality processing of the surface of the diaphragm spring in the area of the bend of the fingers. a) Modeling area - $15^{0}$; b) General view.

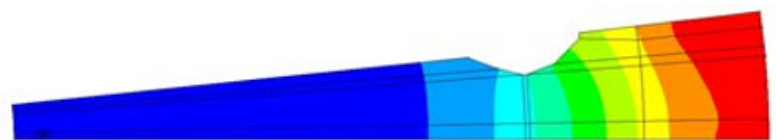

a)

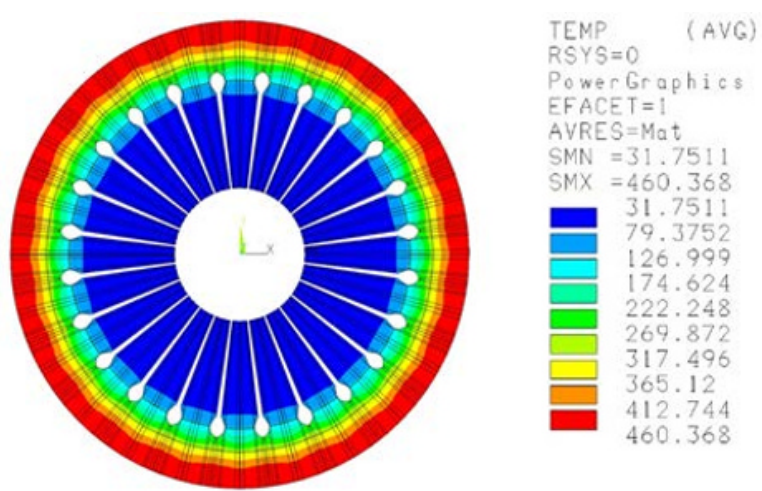

b)

Fig. 9. The final temperature distribution after the heating process completion in a longitudinal magnetic field. The diaphragm spring diameter is $350 \mathrm{~mm}$; the current value is $288 \mathrm{~A}$ : a) Modeling area - 705’; b) General view.

ANSYS package have been developed. The programs automatically perform preliminary initial data preparation, construct a geometric model, assign physical material properties, set boundary conditions, FE meshing, solve the electromagnetic and thermal tasks, and post-process the calculation results. The methodology extends to a number of products with azimuthal periodicity, but has a significant limitation associated with the boundaries of application.

\section{Acknowledgements}

The article was prepared in frame of the project "From engineering knowledge to acceptance of management decision" SPETU "LETI" - the winner of the AllRussian competition of youth projects among higher education educational organizations in 2019 FADM "Rosmolodezh", agreement No. 091-15-2019-069 from 28.08.2019

\section{References}

1. Kuvaldin A. B., Features of mathematical modeling of induction heating of rotary disks of turbine engines,
Actual problems of energy-saving electrical technologies APEET-2014: collection of scientific papers. Yekaterinburg: [UrFU], 2014. - pp. 66-71.

2. Mannanov E., Ermekova M., Mazalov V., Numerical simulation of electrothermal processes during induction tempering of a ring-shaped product. In the collection: Science. Technologies. Innovation. Collection of scientific papers in 10 parts 2017. pp. 128-131.

3. Ermekova M., Mannanov E., Kozulina T., Numerical simulation of an induction system for heating a disk. In the collection: Science. Technologies. Innovation. Collection of scientific papers in 9 parts. Edited by E.G. Gurova. 2015. pp. 12-15.

4. Lepeshkin S.A., The study of loop inductors for modeling and increasing the efficiency of heating rotary discs of gas turbine engines. Collection: Energy And Resource Saving - XXI Century materials of the XIII international scientific and practical Internet conference. State Institution "Oryol Regional Center for Energy Saving". 2015. pp. 84-87.

5. Shepelyakovsky K.Z., Hardening of machine parts by by induction surface heating. - M.: Mechanical Engineering, 1972. pp. 287.

6. Mannanov E.R., Galunin S.A., Nikanorov A.N., Nacke B., Kozulina T.P., Development of induction 
systems for heating of disks, St. Petersburg Polytechnical State University Journal. Physics and Mathematics. 12 (2) (2019) pp. 23-31. DOI: 10.18721/JPM.12202.

7. Numerical modelling of an innovative induction heating technique for aluminium extrusion process / $\mathrm{M}$. Zlobina, B. Nacke, A. Nikanorov, S. Galunin // Proc. of The 3rd international forum on strategic technologies IFOST. Novosibirsk, 2008, pp. 161-164.

8. Numerical investigation of different concepts to control the temperature field at the induction heating of aluminum billets by rotation in superconductive magnets / M. Zlobina, B. Nacke, A. Nikanorov // 6th International conference on Electromagnetic processing of materials “EPM 2009”: Proc. Of Conf., Dresden (Germany), 19-23 October, 2009. / Dresden, 2009. pp. 177-180.

9. Temperature Distribution in Aluminium Billets Heated by Rotation in Static Magnetic Field Produced by Superconducting Magnets / M. Fabbri, A. Morandi, F. Negrini // COMPEL, Vol. 24, n.1, 2005, pp. 281-290.

10. Numerical simulation of induction heating of aluminium billets by rotation in DC magnetic field / B. Nacke, M. Zlobina, A. Nikanorov, A. Ulferts // Proceedings of International Symposium on Heating by Electromagnetic Sources, Padua, 2007, pp. 497-504.

11. Mannanov E.R., Muratov A.A., Galunin S.A., Numerical study of spatial tool for the temperature distribution controlling in rotation heating systems. Research and development of young scientists: A collection of materials of the VI int. scientific-practical Conf., Novosibirsk. Oct 162015 - Novosibirsk: NSTU Publishing House, 2015 . pp. 71-75.

12. Mannanov E.R., Muratov A.A., Galunin S.A. Numerical modeling in heating systems by rotation. Electro. Electrical engineering, power industry, electrical industry. 2016. No. 1. pp. 20-22.

13. Rupnar A., Design and Analysis of Diaphragm Spring of a Single Plate Dry Clutch. IJSTE International Journal of Science Technology \& Engineering, Volume 2, Issue 12, June 2016. pp. 26 - 30. 14. Simulation algorithm for induction heating of rotated workpieces with complex shape / E. Mannanov, S. Galunin, A. Nikanorov, B. Nacke // XVIII International UIE - Congress "Electrotechnologies for Material Processing”, Hannover (Germany), June 6-9, 2017. P. 491-496.

15. Lepeshkin S.A., Modeling and research of temperature distribution in rotating disks with mutual influence. In the collection: Energy And Resource Saving - XXI Century materials XII int. scientificpractical internet conf 2014.pp. 77-79.

16. Numerical model approaches for in-line strip induction heating / S.A. Galunin, M.V. Zlobina, K.Y. Blinov // In the collection: IEEE EUROCON 2009, EUROCON 2009 St. Petersburg, 2009. S. 1607-1610.

17. Numerical optimization of induction through heating for forging / S. Galunin, M. Zlobina, A.Nikanorov, Yu. Blinov // In the collection: Proceedings - 9th RussianKorean International Symposium on Science and Technology, KORUS-2005 9th Russian-Korean International Symposium on Science and Technology,
KORUS-2005. sponsors: Novosibirsk State Technical University. Novosibirsk, 2005. pp. 313-314.

18. Nikanorov A., Schülbe H., Galunin S., From expert solution to optimal design of electrothermal installations. Elektrowaerme International, Edition B: Industrielle Elektrowaerme. 2004. № 4. pp. 177-178.

19. D. Schlesselmann, B.Nacke, A.Nikanorov, S.Galunin , Coupled numerical multiphysics simulation methods in induction surface hardening. In the collection: Coupled Problems 2015 - Proceedings of the 6th International Conference on Coupled Problems in Science and Engineering 6. 2015. pp. 392-403.

20. Kuvaldin A.B., Lepeshkin A.R., Lepeshkin S.A., The heating method and hardening of gears with rotation in a magnetic field. In the collection: Energy And Resource Saving - XXI Century materials XII int. Scientific Pract. internet conf. 2016. pp. 147-151.

21. Kozulina T.P., Mannanov E.R., Galunin S.A., Kudryash M.N., Induction system for hardening camshafts of internal combustion engines. In the collection: 67th scientific and technical. conf. SPbGETU "LETI", St. Petersburg, Jan 27. - Feb 32014 - SPb.: Publishing house of SPbGETU, 2014 - pp. 151-154. 